\title{
Sectorization of Full Kekre's Wavelet Transform for Feature extraction of Color Images
}

\author{
H.B.Kekre \\ Sr. Professor \\ MPSTME, SVKM's NMIMS (Deemed-to be-University) \\ Vile Parle West, Mumbai -56,INDIA \\ hbkekre@yahoo.com
}

\author{
Dhirendra Mishra \\ Associate Professor \& PhD Research Scholar \\ MPSTME, SVKM's NMIMS (Deemed-to be-University) \\ Vile Parle West, Mumbai -56,INDIA \\ dhirendra.mishra@gmail.com
}

\begin{abstract}
An innovative idea of sectorization of Full Kekre's Wavelet transformed (KWT)[1] images for extracting the features has been proposed. The paper discusses two planes i.e. Forward plane (Even plane) and backward plane (Odd plane). These two planes are sectored into 4, 8, 12 and 16 sectors. An innovative concept of sum of absolute difference (AD) has been proposed as the similarity measuring parameters and compared with the well known Euclidean distance (ED).The performances of sectorization of two planes into different sector sizes in combination with two similarity measures are checked. Class wise retrieval performance of all sectors with respect to the similarity measures i.e. ED and AD is analyzed by means of its class (randomly chosen 5 images) average precision- recall cross over points, overall average (average of class average) precisionrecall cross over points and two new parameters i.e. LIRS and LSRR.
\end{abstract}

Keywords- CBIR, Kekre's Wavelet Transform (KWT), Euclidian Distance, Sum of Absolute Difference, LIRS, LSRR, Precision and Recall.

\section{INTRODUCTION}

Content based image retrieval i.e. CBIR [2-6] is well known technology being used and being researched upon for the retrieval of images from the large image databases. CBIR has been proved to be very much needed technology to be researched on due to its applicability in various applications like face recognition, finger print recognition, pattern matching[7][8][9], verification /validation of images etc. The concept of CBIR can be easily understood by the figure 1 as shown below. Every CBIR systems needs functionality for feature extraction of an image viz. shape, color, texture which can represent the uniqueness of the image for the purpose of best match in the database to be searched. The features of the query image are compared with the features of all images in the feature database using various mathematical construct known as similarity measures. These mathematical similarity measuring techniques checks the similarity of features extracted to classify the images in the relevant and irrelevant classes. The research in CBIR needs to be done to explore two aspects first is the better method of feature extraction having maximum components of uniqueness and faster, accurate mathematical models of similarity measures. As the figure 1 shows the example of query image of an horse being provided to CBIR system as query and the images of relevant classes are retrieved. Relevance feedback of the retrieval is used for the machine learning purpose to check the accuracy of the retrieval which in turn helps one to focus on the modification in the current approach to have improved performance.

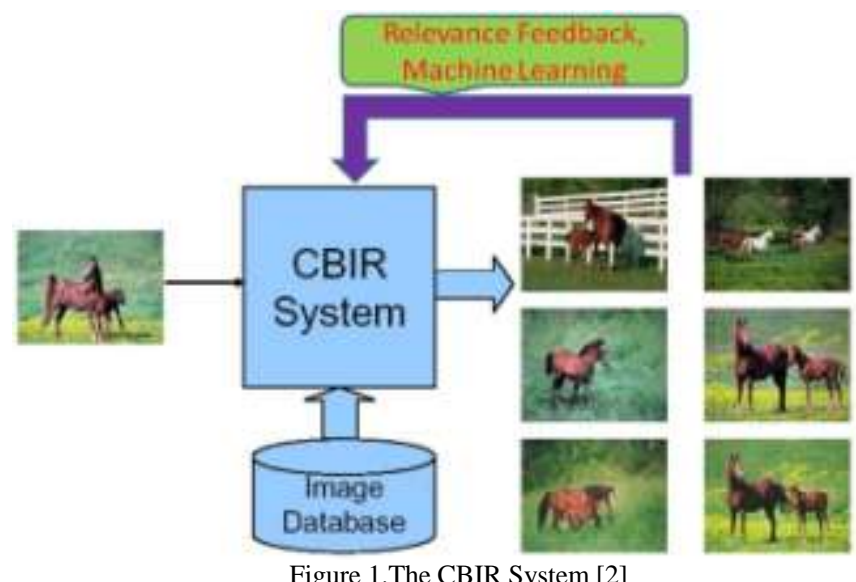

Many researches are currently working on the very open and demanding field of CBIR. These researches focus on to generate the better methodologies of feature extractions in both spatial domain and frequency domain. Some methodologies like block truncation coding [10-11], various transforms: FFT [12-14], Walsh transform [15-21], DCT [22], DST [23] and other approaches like Hashing [24], Vector quantization [25], Contour let transform [5], has already been developed

In this paper we have introduced a novel concept of Sectorization of Full Kekre's Wavelet transformed color images for feature extraction. Two different similarity measures parameters i.e. sum of absolute difference and Euclidean distance are used. Average precision, Recall, LIRS and LSRR are used for performances study of these approaches.

\section{KeKRE'S WAVELET [1]}

Kekre's Wavelet transform is derived from Kekre's transform. From NxN Kekre's transform matrix, we can generate Kekre's Wavelet transform matrices of size $(2 \mathrm{~N}) \mathrm{x}(2 \mathrm{~N}),(3 \mathrm{~N}) \mathrm{x}(3 \mathrm{~N}), \ldots \ldots,(\mathrm{N} 2) \mathrm{x}(\mathrm{N} 2)$. For example, from $5 x$ 5.Kekre's transform matrix, we can generate Kekre's Wavelet transform matrices of size 10x10, 15x15, 20x20 and 
25x25. In general MxM Kekre's Wavelet transform matrix can be generated from NxN Kekre's transform matrix, such that M $=\mathrm{N}^{*} \mathrm{P}$ where $\mathrm{P}$ is any integer between 2 and $\mathrm{N}$ that is, $2 \leq \mathrm{P} \leq$ N. Kekre's Wavelet Transform matrix satisfies $[\mathrm{K}][\mathrm{K}]_{\mathrm{T}}=[\mathrm{D}]$ Where $\mathrm{D}$ is the diagonal matrix this property and hence it is orthogonal. The diagonal matrix value of Kekre's transform matrix of size $\mathrm{NxN}$ can be computed as

$$
D(x, y)=\left\{\begin{array}{cl}
2 & \text {, if } x=y=N \\
N & \text { if } x=y=1 \\
0 & \text {, if } x \neq y \\
D(x+1, y+1)+2(N-x+1) & \text { if } x=y=p \text { and } p \neq 1 \text { or } N
\end{array}\right.
$$

\section{PLANE FORMATION AND ITS SECTORIZATION}

$$
\text { [12-19],[22-23] }
$$

The components of Full KWT transformed image shown in the red bordered area (see Figure 2) are used to generate feature vectors. The average of zeoeth row, column and last row and column components are augmented to feature vector generated. Color codes are used to differentiate between the co-efficients plotted on Forward (Even) plane as light red and light blue for co-efficients belonging to backward (Odd) plane. The coefficient with light red background i.e. at position $(1,1),(2,2) ;(1,3),(2,4)$ etc. are taken as X1 and Y1 respectively and plotted on Even plane. The co-efficient with light blue background i.e. at position $(2,1),(1,2) ;(2,3),(1,4)$ etc. are taken as $\mathrm{X} 2$ and $\mathrm{Y} 2$ respectively and plotted on Odd plane.

\begin{tabular}{|r|l|l|l|l|l|l|}
\hline \multicolumn{2}{|c|}{0} & \multicolumn{2}{|c|}{1} & \multicolumn{2}{c|}{3} & \multicolumn{2}{|c|}{4} \\
\hline & 0,0 & 0,1 & 0,2 & 0,3 & 0,4 & 0,5 \\
\hline 1 & 1,0 & 1,1 & 1,2 & 1,3 & 1,4 & 1,5 \\
\hline 2 & 2,0 & 2,1 & 2,2 & 2,3 & 2,4 & 2,5 \\
\hline 3 & 3,0 & 3,1 & 3,2 & 3,3 & 3,4 & 3,5 \\
\hline 4 & 4,0 & 4,1 & 4,2 & 4,3 & 4,4 & 4,5 \\
\hline 5 & 5,0 & 5,1 & 5,2 & 5,3 & 5,4 & 5,5 \\
\hline
\end{tabular}

Figure 2: KWT component arrangement in an Transformed Image.

Even plane of Full KWT is generated with taking KWT components into consideration as all $\mathrm{X}(\mathrm{i}, \mathrm{j}), \mathrm{Y}(\mathrm{i}+1, \mathrm{j}+1)$ components for even plane and all $\mathrm{X}(\mathrm{i}+1, \mathrm{j}), \mathrm{Y}(\mathrm{i}, \mathrm{j}+1)$ components for odd plane as shown in the Figure 3. Henceforth for our convenience we will refer $\mathrm{X}(\mathrm{i}, \mathrm{j})=\mathrm{X} 1, \mathrm{Y}(\mathrm{i}+1, \mathrm{j}+1)=\mathrm{Y} 1$ and $\mathrm{X}(\mathrm{i}+1, \mathrm{j})=\mathrm{X} 2$ and $\mathrm{Y}(\mathrm{i}, \mathrm{j}+1)=\mathrm{Y} 2$.

\begin{tabular}{|ll|}
\hline$X(i, j)$ & $Y(i, j+1)$ \\
$X(i+1, j)$ & $Y(i+1, j+1)$ \\
\hline
\end{tabular}

Figure 3: Snapshot of Components considered for Even/Odd Planes.

As shown in the Figure 3 the Even plane of Full KWT considers X1 i.e. all light red background cells $(1,1)$, $(2,2),(1,3),(2,4)$ etc. on $\mathrm{X}$ axis and $\mathrm{Y} 1$ i.e. $(1,2),(2,1),(1,4),(2,3)$ etc. on Y axis. The Odd plane of Full KWT considers X1 i.e. all light blue background cells $(1,2),(2,1),(1,4),(2,3)$ etc. on $X$ axis and $\mathrm{Y} 1$ i.e. $(1,2),(2,1),(1,4),(2,3)$ etc. on $\mathrm{Y}$ axis.

\section{RESULTS AND DISCUSSION.}

Augmented Wang image database [4] has been used for the experiment. The database consists of 1055 images of 12 different classes such as Flower, Sunset, Barbie, Tribal, Cartoon, Elephant, Dinosaur, Bus, Scenery, Monuments, Horses, Beach. Class wise distribution of all images in the database has been shown in the Figure 7.

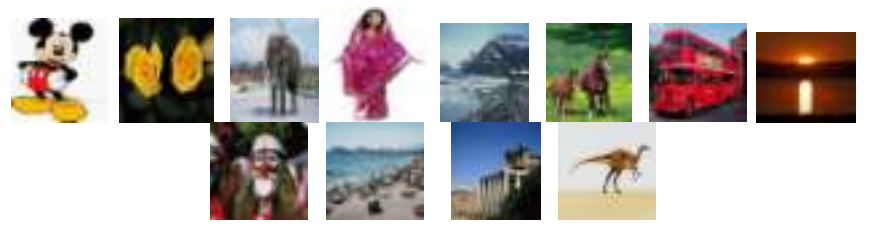

Figure 7: Class wise distribution of images in the Image database consists of Sunset:51, Cartoon:46,

Flower:100,Elephants:100,Barbie:59,Mountains:100,Horse:100,Bus:100,Triba 1:100,Beaches:99,Monuments:100,Dinasour :100

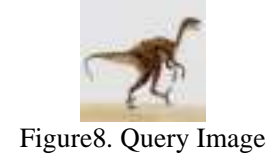

The query image of the class dinosaur has been shown in Figure 8. For this query image the result of retrieval of both approaches of Full KWT wavelet transformed image sectorization of even and odd planes. The Figure 9 shows First 20 Retrieved Images sectorization of Full KWT wavelet Forward (Even) plane (16 Sectors) with sum of absolute difference as similarity measure. There are two retrieval from irrelevant class.

The first irrelevant image occurred $15^{\text {th }}$ position and second on $20^{\text {th }}$ position (shown with red boundary) in the even plane sectorization. The result of odd plane sectorization shown in Figure 10; the retrieval of first 20 images containing 2 irrelevant retrievals but the first irrelevant class has occurred at $17^{\text {th }}$ position.

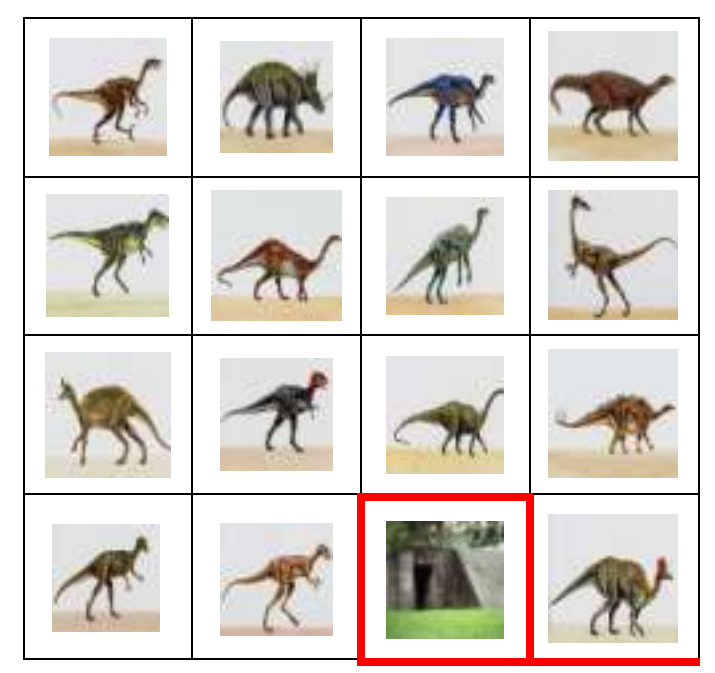




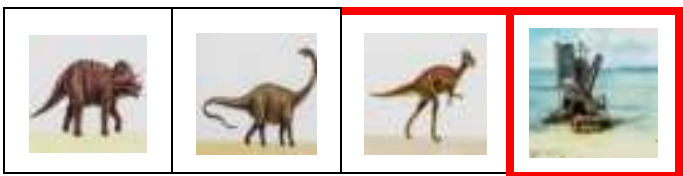

Figure 9: First 20 Retrievals of Full KWT Forward (Even) plane sectorization into 16 Sectors with sum of absolute difference as similarity measure.

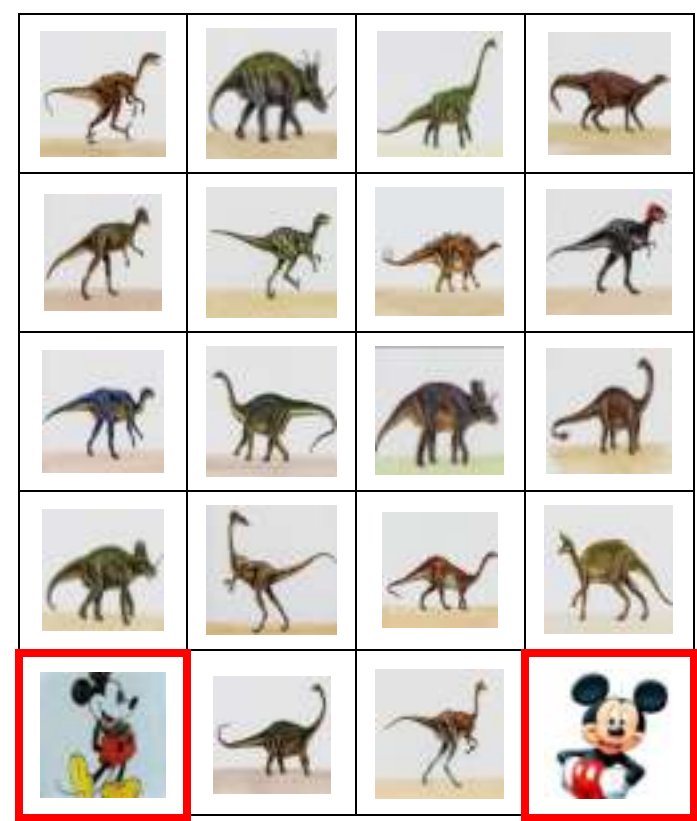

Figure 10: First 20 Retrievals of Full KWT Backward (Odd) plane sectorization into 16 Sectors with sum of absolute difference as similarity measure..

Feature database includes feature vectors of all images in the database. Five random query images of each class were used to search the database. The image with exact match gives minimum sum of absolute difference and Euclidian distance. To check the effectiveness of the work and its performance with respect to retrieval of the images we have calculated the overall average precision and recall and its cross over values and plotted class wise. The Equations (2) and (3) are used for precision and recall calculation whilst two new parameters i.e. LIRS (Length of initial relevant string of images) and LSRR (Length of string to recover all relevant images) are used as shown in Equations (4) and (5).

$$
\begin{aligned}
& \text { Precision }=\frac{\text { Number of Releyant inages Retritved }}{\text { Total Number of Images Retriered }} \\
& \text { Recall }=\frac{\text { Number of Relavant Lmages Retriered }}{\text { Totat Nuewber of Releyant Images in the Dotabase }}
\end{aligned}
$$

$$
\text { LRS }=\frac{\text { Length of Initial Relevant string of Images }}{\text { Total Relevent lmages Retrieved }}
$$

$$
\text { LSRR }=\frac{\text { Lenght of String to Recover all Retarent Inagts }}{\text { Totel Images in the Datsbase }}
$$

All these parameters lie between $0-1$ hence they can be expressed in terms of percentages. The newly introduced parameters give the better performance for higher value of LIRS and Lower value of LSRR [8-13].

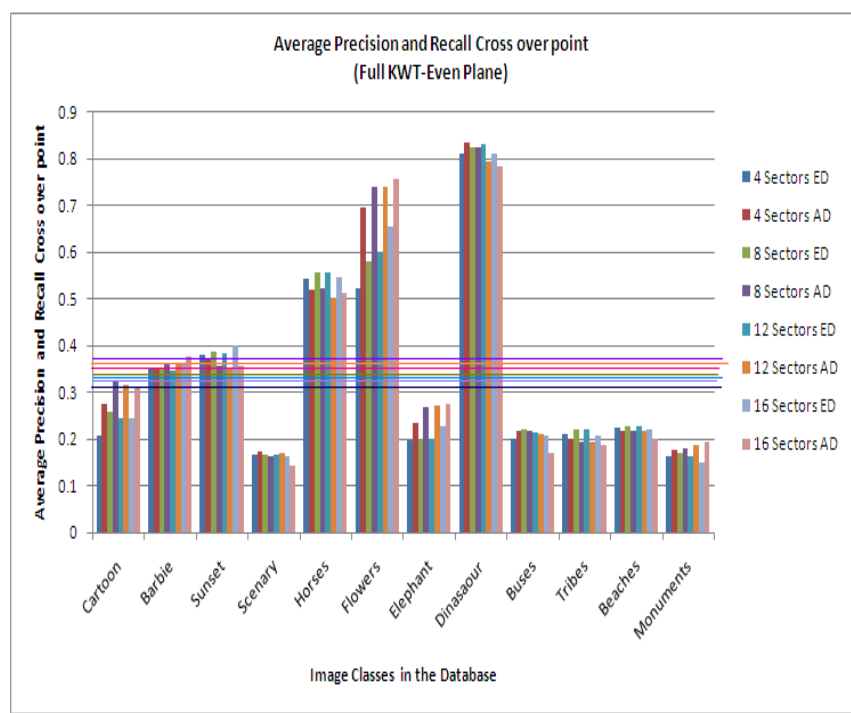

Figure 11: Class wise Average Precision and Recall cross over points of

Forward Plane (Even) sectorization of Full KWT Wavelet with sum of Absolute Difference (AD) and Euclidean Distance (ED) as similarity measure. 


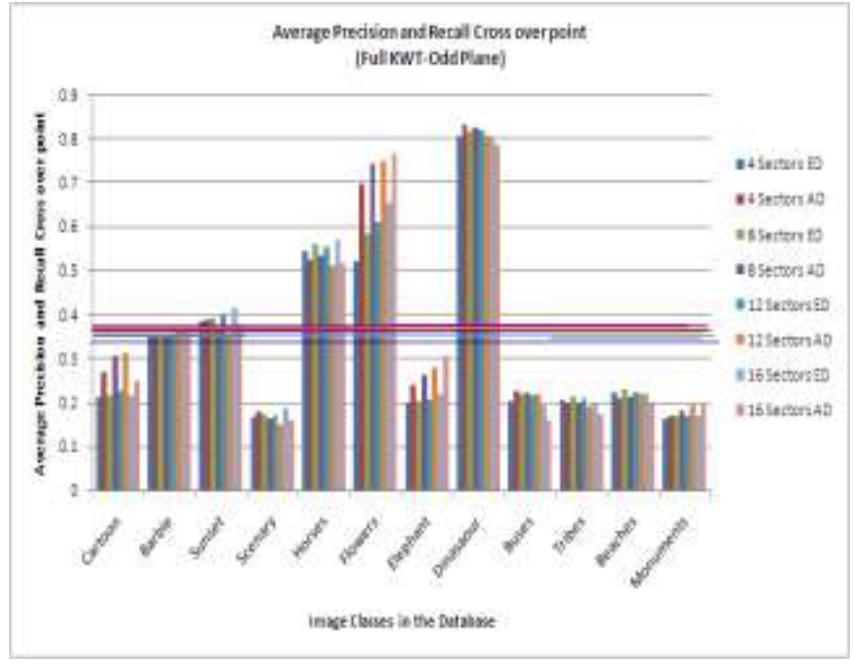

Figure 12: Class wise Average Precision and Recall cross over points of Backward Plane (Odd) sectorization of Full KWT Wavelet with Absolute Difference (AD) and Euclidean Distance (ED) as similarity measure.

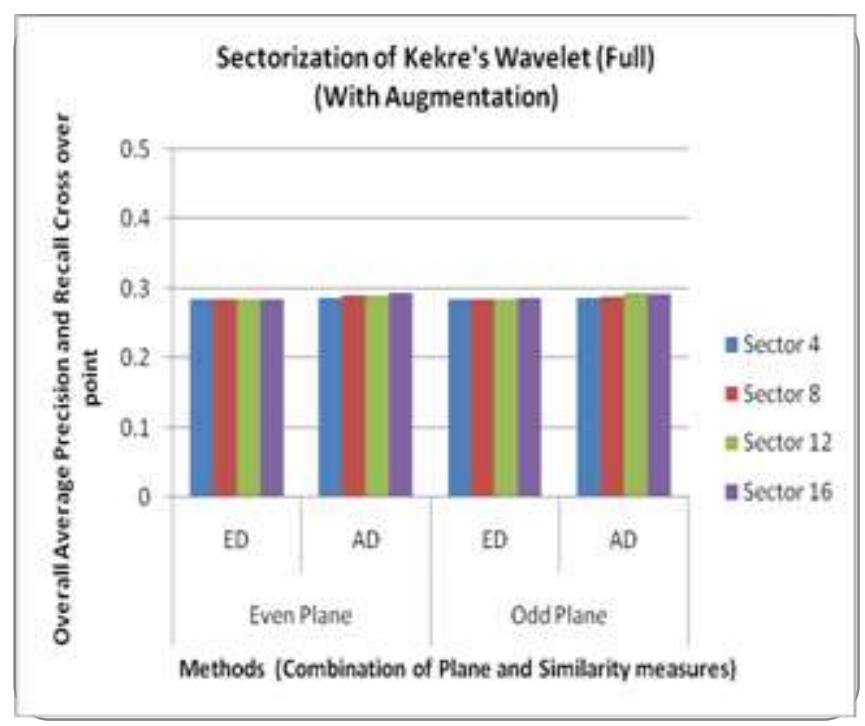

Figure 13: Comparison of Overall Precision and Recall cross over points of sectorization of Full KWT Wavelet with sum of Absolute Difference (AD) and Euclidean Distance (ED) as similarity measure.

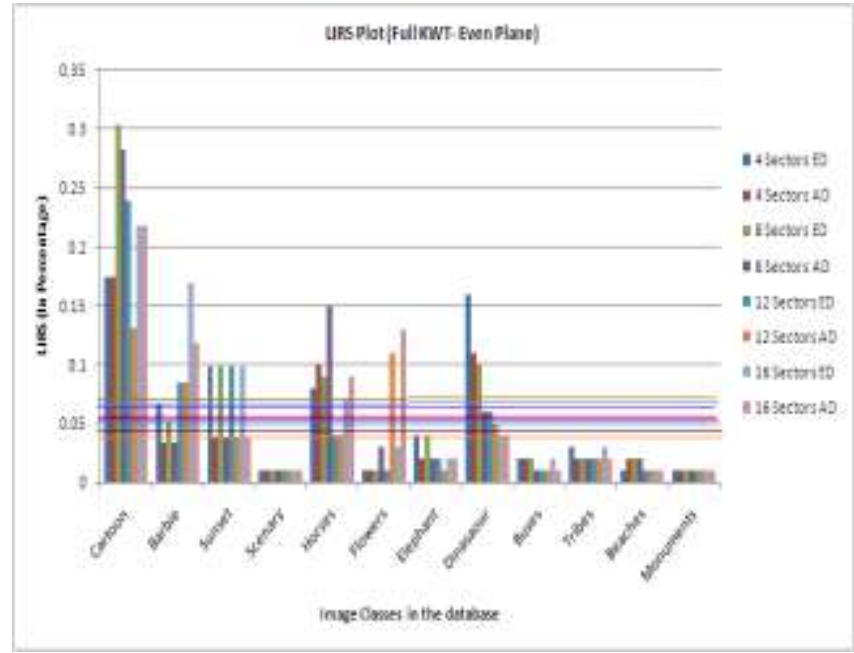

Figure 14: The LIRS Plot of sectorization of forward plane of Full KWT transformed images . Overall Average LIRS performances (Shown with Horizontal lines :0.082 (4 Sectors ED), 0.052 (4 Sectors AD), 0.071(8 Sectors ED), 0.051(8 Sectors AD), 0.075(12 Sectors ED), 0.069(12 Sectors AD), 0.053(16 Sectors ED), 0.053(16 Sectors AD) ).

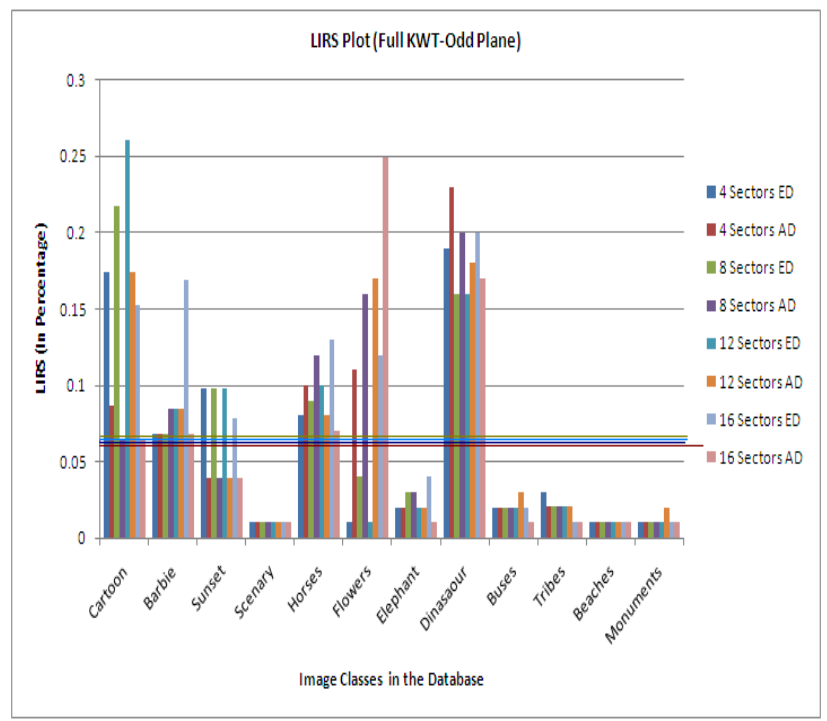

Figure 15: The LIRS Plot of sectorization of Backward plane of Full KWT transformed images . Overal Average LIRS performances (Shown with Horizontal lines :0.081 (4 Sectors ED), 0.054 (4 Sectors AD), 0.073(8 Sectors ED), 0.050(8 Sectors AD), 0.064(12 Sectors ED), 0.049(12 Sectors AD), 0.056(16 Sectors ED), 0.042(16 Sectors AD) ). 


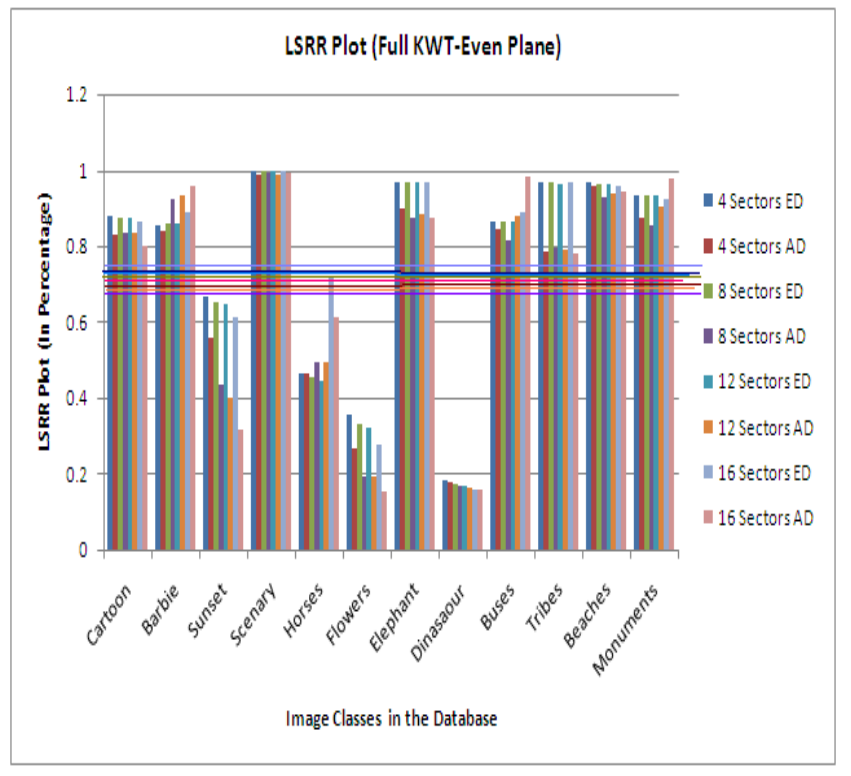

Figure 16: The LSRR Plot of sectorization of forward plane of Full KWT transformed images . Overall Average LSRR performances (Shown with Horizontal lines :0.77 (4 Sectors ED), 0.71 (4 Sectors AD), 0.76(8 Sectors ED), 0.71(8 Sectors AD), 0.76(12 Sectors ED), 0.73(12 Sectors AD), 0.74(16 Sectors ED), 0.71(16 Sectors AD) ).

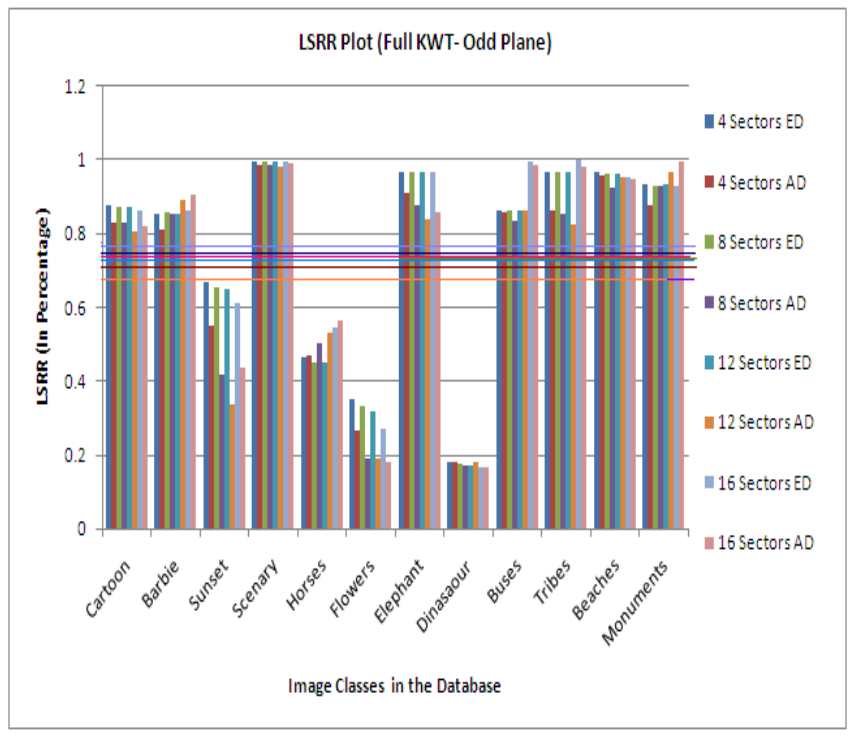

Figure 17: The LSRR Plot of sectorization of backward plane of Full KWT transformed images . Overall Average LSRR performances (Shown with Horizontal lines :0.77(4 Sectors ED), 0.729 (4 Sectors AD), $0.76(8$ Sectors ED), 0.725(8 Sectors AD), 0.756(12 Sectors ED), 0.726(12 Sectors AD), 0.759(16 Sectors ED), 0.727(16 Sectors AD) ).

\section{CONCLUSION}

The work experimented on 1055 image database of 12 different classes discusses the performance of sectorization of Full KWT wavelet transformed color images for image retrieval. The work has been performed with both approaches of sectorization of forward (even) plane and backward (odd) planes. The performance of the methods proposed checked with respect to various sector sizes and similarity measuring approaches viz. Euclidian distance and sum of absolute difference. We calculated the average precision and recall cross over point of 5 randomly chosen images of each class and the overall average is the average of these averages. The observation is that sectorization of both planes of full KWT wavelet transformed images give less than $30 \%$ of the overall average retrieval of relevant images as shown in the Figure 13. The class wise plot of these average precision and recall cross over points as shown in Figure 11 and Figure 12 for both approaches depicts that the retrieval performance varies from class to class and from method to method wherein horses, flower and dinosaur classes have retrieval more than $50 \%$. They have the performance above the average of all methods as shown by horizontal lines. New parameter LIRS and LSRR gives good platform for performance evaluation to judge how early all relevant images is being retrieved (LSRR) and it also provides judgement of how many relevant images are being retrieved as part of first set of relevant retrieval (LIRS).The value of LIRS must be minimum and LSRR must be minimum for the particular class if the overall precision and recall cross over point of that class is maximum. This can be clearly seen in Figures 14 to Figure 17. This observation is very clearly visible for dinosaur class however the difference of LIRS and LSRR of other classes varies. The sum of absolute difference as similarity measure is recommended due to its lesser complexity and better retrieval rate performance compared to Euclidian distance.

\section{REFERENCES}

[1] H.B.Kekre, Archana Athawale and Dipali sadavarti, "Algorithm to generate Kekre's Wavelet transform from Kekre's Transform", International Journal of Engineering, Science and Technology, Vol.2No.5,2010 pp.756-767.

[2] Dr. Qi, "semantic based CBIR(content based image retrieval)",http://cs.usu.edu/htm/REU-Current-Projects.

[3] Kato, T., "Database architecture for content based image retrieval in Image Storage and Retrieval Systems" (Jambardino A and Niblack W eds),Proc SPIE 2185, pp 112-123, 1992.

[4] Ritendra Datta,Dhiraj Joshi,Jia Li and James Z. Wang, “ Image retrieval:Idea,influences and trends of the new age",ACM Computing survey,Vol 40,No.2,Article 5,April 2008.

[5] Ch.srinivasa rao,S. srinivas kumar,B.N.Chaterjii, "content based image retrieval using contourlet transform", ICGST-GVIP Journal, Vol.7 No. 3, Nov2007.

[6] John Berry and David A. Stoney "The history and development of fingerprinting," in Advances in Fingerprint Technology, Henry C. Lee and R. E. Gaensslen, Eds., pp. 1-40. CRC Press Florida, $2^{\text {nd }}$ edition, 2001

[7] Arun Ross, Anil Jain, James Reisman, "A hybrid fingerprint matcher," Int'l conference on Pattern Recognition (ICPR), Aug 2002.

[8] A. M. Bazen, G. T. B.Verwaaijen, S. H. Gerez, L. P. J. Veelenturf, and B. J. van der Zwaag, "A correlation-based fingerprint verification system," Proceedings of the ProRISC2000 Workshop on Circuits, Systems and Signal Processing, Veldhoven, Netherlands, Nov 2000.

[9] H.B.Kekre, Tanuja K. Sarode, Sudeep D. Thepade, "DST Applied to Column mean and Row Mean Vectors of Image for Fingerprint Identification", International Conference on Computer Networks and Security, ICCNS-2008, 27-28 Sept 2008, Vishwakarma Institute of Technology, Pune.

[10] H.B.Kekre, Sudeep D. Thepade, "Using YUV Color Space to Hoist the Performance of Block Truncation Coding for Image Retrieval", IEEE International Advanced Computing Conference 2009 (IACC'09), Thapar University, Patiala, INDIA, 6-7 March 2009. 
[11] H.B.Kekre, Sudeep D. Thepade, "Image Retrieval using Augmented Block Truncation Coding Techniques", ACM International Conference on Advances in Computing, Communication and Control (ICAC3-2009), pp.: 384-390, 23-24 Jan 2009, Fr. Conceicao Rodrigous College of Engg., Mumbai. Available online at ACM portal.

[12] H. B. Kekre, Dhirendra Mishra, "Digital Image Search \& Retrieval using FFT Sectors" published in proceedings of National/Asia pacific conference on Information communication and technology(NCICT 10) $5^{\mathrm{TH}} \& 6^{\mathrm{TH}}$ March 2010.SVKM'S NMIMS MUMBAI

[13] H.B.Kekre, Dhirendra Mishra,"Digital Image Search \& Retrieval using FFT Sectors of Color Images" published in International Journal of Computer Science and Engineering (IJCSE) Vol. 02,No.02,2010,pp.368-372 ISSN 0975-3397 available online at http://www.enggjournals.com/ijcse/doc/IJCSE10-02- 02-46.pdf

[14] H.B.Kekre, Dhirendra Mishra, "CBIR using upper six FFT Sectors of Color Images for feature vector generation" published in International Journal of Engineering and Technology(IJET) Vol. 02, No. 02, 2010, 49-54 ISSN 0975-4024 available online at http://www.enggjournals.com/ijet/doc/IJET10-02- 02-06.pdf

[15] H.B.Kekre, Dhirendra Mishra, "Four walsh transform sectors feature vectors for image retrieval from image databases", published in international journal of computer science and information technologies (IJCSIT) Vol. 1 (2) 2010, 33-37 ISSN 0975-9646 available online at http://www.ijcsit.com/docs/vol1issue2/ijcsit2010010201.pdf

[16] H.B.Kekre, Dhirendra Mishra, "Performance comparison of four, eight and twelve Walsh transform sectors feature vectors for image retrieval from image databases", published in international journal of Engineering, science and technology(IJEST) Vol.2(5) 2010, 1370-1374 ISSN 0975-5462 available online at http://www.ijest.info/docs/IJEST10-02-05-62.pdf

[17] H.B.Kekre, Dhirendra Mishra, “ density distribution in walsh transfom sectors ass feature vectors for image retrieval", published in international journal of compute applications (IJCA) Vol.4(6) 2010, 30-36 ISSN 0975-8887 available online http://www.ijcaonline.org/archives/volume4/number6/829-1072

[18] H.B.Kekre, Dhirendra Mishra, "Performance comparison of density distribution and sector mean in Walsh transform sectors as feature vectors for image retrieval", published in international journal of Image Processing (IJIP) Vol.4(3) 2010, ISSN 1985-2304 available online at http://www.cscjournals.org/csc/manuscript/Journals/IJIP/Volume4/Issue 3/IJIP-193.pdf

[19] H.B.Kekre, Dhirendra Mishra, "Density distribution and sector mean with zero-sal and highest-cal components in Walsh transform sectors as feature vectors for image retrieval", published in international journal of Computer scienece and information security (IJCSIS) Vol.8(4) 2010, ISSN 1947-5500 available online http://sites.google.com/site/ijcsis/vol8-no-4-jul-2010

[20] H.B.Kekre, Vinayak Bharadi, "Walsh Coefficients of the Horizontal \& Vertical Pixel Distribution of Signature Template", In Proc. of Int. Conference ICIP-07, Bangalore University, Bangalore. 10-12 Aug 2007.

[21] J. L. Walsh, "A closed set of orthogonal functions" American Journal of Mathematics, Vol. 45, pp.5-24,year 1923.

[22] H.B.Kekre, Dhirendra Mishra, "DCT sectorization for feature vector generation in CBIR", International journal of computer application (IJCA),Vol.9, No.1,Nov.2010,ISSN:1947-5500 http://ijcaonline.org/archives/volume9/number1/1350-1820

[23] H.B.Kekre, Dhirendra Mishra, "DST Sectorization for feature vector generation", Universal journal of computer science and engineering technology(Unicse),Vol.1,No.1Oct 2010 available at http://www.unicse.oeg/index.php?option=com content and view $=$ article \&id $=54 \&$ itemid $=27$

[24] H.B.Kekre, Dhirendra Mishra, "Content Based Image Retrieval using Weighted Hamming Distance Image hash Value" published in the proceedings of international conference on contours of computing technology pp. 305-309 (Thinkquest2010) 13th \& 14 ${ }^{\text {th }}$ March 2010.

[25]H.B.Kekre, Tanuja K. Sarode, Sudeep D. Thepade, "Image Retrieval using Color-Texture Features from DST on VQ Codevectors obtained by Kekre's Fast Codebook Generation", ICGST International Journal on Graphics, Vision and Image Processing (GVIP), Available online at http://www.icgst.com/gvip

\section{AUTHORS PROFILE}

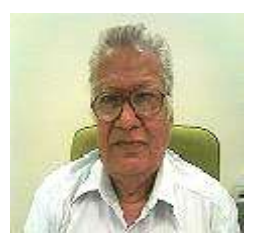

H. B. Kekre has received B.E. (Hons.) in Telecomm. Engg. from Jabalpur University in 1958, M.Tech (Industrial Electronics) from IIT Bombay in 1960, M.S.Engg. (Electrical Engg.) from University of Ottawa in 1965 and Ph.D.(System Identification) from IIT Bombay in 1970. He has worked Over 35 years as Faculty and H.O.D. Computer science and Engg. At IIT Bombay. From last 13 years working as a professor in Dept. of Computer Engg. at Thadomal Shahani Engg. College, Mumbai. He is currently senior Professor working with Mukesh Patel School of Technology Management and Engineering, SVKM's NMIMS University vile parle west Mumbai. He has guided 17 PhD.s 150 M.E./M.Tech Projects and several B.E./B.Tech Projects. His areas of interest are Digital signal processing, Image Processing and computer networking. He has more than 350 papers in National/International Conferences/Journals to his credit. Recently ten students working under his guidance have received the best paper awards. Two research scholars working under his guidance have been awarded $\mathrm{Ph}$. D. degree by NMIMS University. Currently he is guiding $10 \mathrm{PhD}$. Students. He is life member of ISTE and Fellow of IETE.

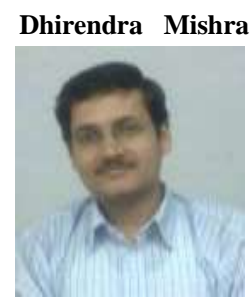

has received his BE (Computer Engg) and M.E. (Computer Engg) degree from University of Mumbai, Mumbai, India $\mathrm{He}$ is $\mathrm{PhD}$ Research Scholar and working as Associate Professor in Computer Engineering department of Mukesh Patel School of Technology Management and Engineering, SVKM's NMIMS University, Mumbai, India. He is life member of Indian Society of Technical education (ISTE), Member of International association of computer science and information technology (IACSIT), Singapore, Member of International association of Engineers (IAENG). His areas of interests are Image Processing, Image Databases; Pattern matching, Operating systems, Information Storage and Management. 